\title{
Measurement of temperature inside die and estimation of interfacial heat transfer coefficient in squeeze casting
}

\author{
Fei-fan Wang ${ }^{1,2}$, Ke-yan Wu ${ }^{1,2}$, Xu-yang Wang ${ }^{1,2}$, and *Zhi-qiang Han ${ }^{1,2}$ \\ 1. School of Materials Science and Engineering, Tsinghua University, Beijing 100084, China \\ 2. Key Laboratory for Advanced Materials Processing Technology (Ministry of Education), Tsinghua University, Beijing 100084, China
}

\begin{abstract}
As an advanced near-net shape technology, squeeze casting is an excellent method for producing high integrity castings. Numerical simulation is a very effective method to optimize squeeze casting process, and the interfacial heat transfer coefficient (IHTC) is an important boundary condition in numerical simulation. Therefore, the study of the IHTC is of great significance. In the present study, experiments were conducted and a "plate shape" aluminum alloy casting was cast in $\mathrm{H} 13$ steel die. In order to obtain accurate temperature readings inside the die, a special temperature sensor units (TSU) was designed. Six $1 \mathrm{~mm}$ wide and $1 \mathrm{~mm}$ deep grooves were machined in the sensor unit for the placement of the thermocouples whose tips were welded to the end wall. Each groove was machined to terminate at a particular distance $(1,3$, and $6 \mathrm{~mm})$ from the front end of the sensor unit. Based on the temperature measurements inside the die, the interfacial heat transfer coefficient (IHTC) at the metal-die interface was determined by applying an inverse approach. The acquired data were processed by a low pass filtering method based on Fast Fourier Transform (FFT). The feature of the IHTC at the metal-die interface was discussed.
\end{abstract}

Key words: squeeze casting; interfacial heat transfer coefficient; temperature sensor unit; inverse approach

CLC numbers: TP391.99

Document code: $\mathrm{A}$

Article ID: 1672-6421(2017)05-327-06

\begin{abstract}
L ightweight components have vital importance due to weight reduction in vehicles because of high strength-to-weight ratio. The utilization of these, whose materials are mainly aluminum and magnesium alloys, in the automotive industry has therefore significantly increased in the past few years ${ }^{[1]}$. However, the mechanical properties of these components are often deteriorated by defects such as shrinkage and gas pores due to inappropriate casting technologies ${ }^{[2-4]}$. Thus, it is very important to find an appropriate casting technology. Squeeze casting (SC) is a promising near net-shape processing technique, where the molten metal solidifies under a relatively high external pressure, and the fabricated components are normally free of shrinkage and gas pores ${ }^{[5]}$.

In the squeeze casting process, process parameters
\end{abstract}

\section{*Zhi-qiang Han}

Male, born in 1968, Ph.D., Associate Professor. His research interests mainly focus on high integrity and near net shape casting technology for lightweight metal alloys, aluminum and magnesium alloys squeeze casting process and its macro and microscopic modeling, multi-scale and multi-discipline modeling and computation of solidification and casting processes.

E-mail: zqhan@tsinghua.edu.cn

Received: 2017-07-31; Accepted: 2017-08-27 have great influence on the quality of the cast product. Thus, before the full economic and applicable squeeze casting can be achieved in industry, the squeeze casting process parameters should be optimized by employing experiments or numerical simulation. In recent years, with the development of computer technology, numerical simulation has received widespread concerns due to the shorter product development cycle and lower cost. As an important boundary condition in numerical simulation, an accurate interfacial heat transfer coefficient (IHTC) is needed. However, most researchers used the IHTC which was constant or proportional to the applied pressure in their simulations ${ }^{[6]}$. These assumptions were very different from the actual situation of the castingdie interface heat transfer. For example, Lee et $\mathrm{al}^{[7]}$ and Zhu et al. ${ }^{[8-10]}$ proposed models of the IHTC which took the influence of the pressure and the gap at the castingdie interface into account in their simulations based on empirical data. That is to say that the models lacked experimental data support.

While numerous investigations have taken place in the past three decades seeking to experimentally determine the IHTC for different casting processes, there are limited studies concerning the IHTC in squeeze casting. Some researchers investigated the influence of 
the applied pressure on the IHTC by conducting squeeze casting experiments ${ }^{[11-13]}$. Sun et al. ${ }^{[14-15]}$ determined the IHTC of a fivestep-shape casting in magnesium alloy AM60 and aluminum alloy ADC443 and measured the pressure at the casting-die interface using a Kistler pressure transducer. However, all the above-mentioned studies of the IHTC in squeeze casting ignored a very important factor which has great influence on the accurate determination of the IHTC. In the squeeze casting process, the heat flux at the casting-die interface increases dramatically at the pouring moment and the pressure applying moment. At this time, it is very difficult to determine the IHTC accurately.

In this paper, in order to obtain an accurate IHTC, squeeze casting experiments were carried out where a special temperature sensor unit (TSU) was designed to obtain accurate temperature readings inside the die. The acquired data were processed by a low pass filtering method based on Fast Fourier Transform (FFT).

\section{Experiment procedure}

In these experiments, the direct squeeze casting (DSC) process was adopted. A "plate shape" aluminum alloy casting was cast in H13 steel die. Figure 1 shows the plate shape casting with dimensions of $140 \mathrm{~mm} \times 90 \mathrm{~mm} \times 30 \mathrm{~mm}$.
As mentioned in our previous work ${ }^{[16]}$, for the process in which the heat flux at the casting-die interface increased dramatically, in order to accurately determine the IHTC, the nearest distance between the temperature measurement point and the casting-die interface must be smaller than $1 \mathrm{~mm}$. As a result, a special temperature sensor unit was designed. Figure 1 shows the configuration of the temperature sensor unit. Six $1 \mathrm{~mm}$ wide and $1 \mathrm{~mm}$ deep grooves were machined in the sensor unit for the placement of the thermocouples. Each groove was machined to terminate at a particular distance $(1,3$, and $6 \mathrm{~mm})$ from the front end of the sensor unit. The thermocouple tip was welded to the end wall of the groove by stored-energy welding in order to maintain a perfect contact with the temperature measurement point. In order to prevent the welded thermocouples from falling off, high temperature resistant inorganic adhesive was filled in the grooves. As the adhesive very easily adheres to the surface of the temperature sensor unit, the surface needed to be polished before installing the temperature sensor unit to the die. Due to the polishing, the temperature sensor unit must be 0.01 $\mathrm{mm}$ larger than $12_{0}^{+0.018} \mathrm{~mm}$ to prevent the leakage of aluminum.

The temperature sensor unit was manufactured using the same material as the die to ensure that the heat transfer process would not be distorted.
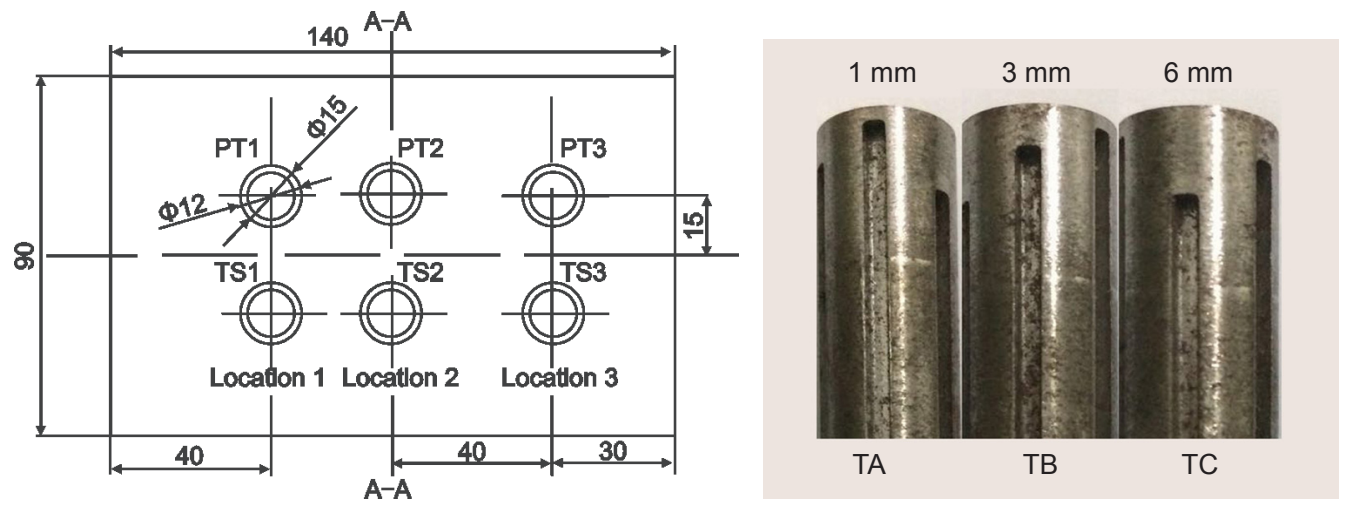

Fig. 1: Configuration of casting and temperature sensor unit

The thermocouples purchased from Omga Engineering Inc. were K-type thermocouples with a thermocouple wire diameter of $0.25 \mathrm{~mm}$. The response time of these thermocouples is about $10 \mathrm{~ms}$.

Figure 1 shows the configuration of casting and installation of the measure unit. Three temperature sensor units, designated TS1-TS3, were installed into the lower die at different locations. Each temperature sensor unit was adjusted into the die until the front wall of the sensor unit approached the cavity surface.

A $1000 \mathrm{kN}$ laboratory hydraulic press and a mid-frequency induction furnace were used for these experiments. The die material was H13 steel. The die coating used in these experiments was based on water and graphite which was sprayed manually onto the surface of the die. A data acquisition system manufactured by Integrated Measurement Corporation
(IMC, Berlin, Germany) was used during the experiment with a sampling frequency of $200 \mathrm{~Hz}$.

Before pouring, the dies were pre-heated to $483 \mathrm{~K}\left(210^{\circ} \mathrm{C}\right)$ using four heat cartridges installed inside the lower die. The experimental procedure included measuring the temperature of the pouring molten aluminum alloy using a sheathed thermocouple which was inserted into the aluminum melt manually, pouring the melt into the lower die, closing the dies, applying and maintaining the pressure, opening the dies, pushing out the casting with ejection mechanism. Every time, after pushing out the casting, the temperature of the die decreased quickly. When all the readings of the temperature sensor units became almost the same and the temperature decreasing rate was below $2{ }^{\circ} \mathrm{C} \cdot \mathrm{min}^{-1}$, the temperature of the die was defined as the initial die temperature. 


\section{Method description}

\subsection{Data processing}

In the field of industrial production, physical quantities, such as temperature, pressure and vibration, are converted to electrical signals by sensors and these electrical signals are collected by the data acquisition system. Due to the complexity of the scene and the intrinsic properties of the measure unit, the undesired noise interference is inevitably carried into the actual measurement data. Usually the noise components of the data have small amplitude and high frequency. In comparison with the temperature and pressure variation trends that would be investigated in the squeeze casting process, the highfrequency noise components in the temperature and pressure measurements caused by temperature and pressure fluctuation or the intrinsic properties of the measure unit were undesired. However, due to the high sampling frequency of the data acquisition system which was set at $200 \mathrm{~Hz}$, the high-frequency noise components (usually $40-50 \mathrm{~Hz}$ ) in the temperature and pressure measurements were also measured and inevitably included in the acquired data. In order to eliminate the negative effect of these high-frequency noise components on the analysis of the IHTC, a low pass filtering method based on Fast Fourier
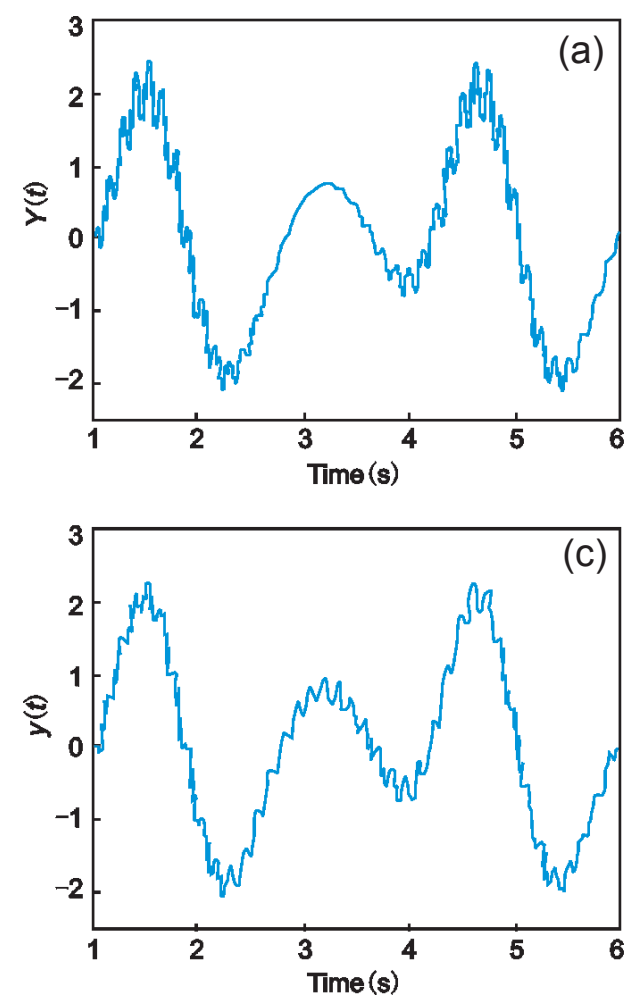

Fig. 2: Low pass filtering method based on FF by $7.96 \mathrm{~Hz}$; (d) filtered by $7.7 \mathrm{~Hz}$

According to the noise frequency, three different types of frequency were chosen as the cutoff frequency for data processing using the low pass filtering method. The first type of the cutoff frequency was $9 \mathrm{~Hz}$, which was higher than the noise frequency. Figure 2(b) shows the result of data processing. The curve shown in Fig. 2(b) was almost the same as the one in Fig.
Transform (FFT) was adopted to process the data.

Fast Fourier Transform (FFT) is an algorithm which can rapidly compute the discrete Fourier transform of a sequence or its inverse. The low pass filtering method based on FFT is a data process method which transforms the data in time domain to frequency domain using FFT algorithm, then eliminates the high-frequency noise components whose frequency is above the cutoff frequency and finally converts the processed data in frequency domain to time domain. The cutoff frequency is the key parameter of the low pass filtering method based on FFT. With a correctly selected cutoff frequency, the low pass filtering method can eliminate the high-frequency noise components without affecting the accuracy of the data. Therefore, it is very important to choose the correct cutoff frequency.

In order to find a way to determine the cutoff frequency, a function was created, as shown by Eq. (1). This function contained a high-frequency noise term, $0.4 \sin t * \sin 50 t$, and the frequency of this noise term was $7.96 \mathrm{~Hz}$. The frequency of other terms in the created function was much lower than the noise frequency. The curve in Fig. 2(a) shows the variation trend of the created function in the range of 1-6 s.

$$
y(t)=\mathrm{e}^{-(\cos t)^{2}}(\sin 2 t+2 \cos 4 t+0.4 \sin t * \sin 50 t)
$$
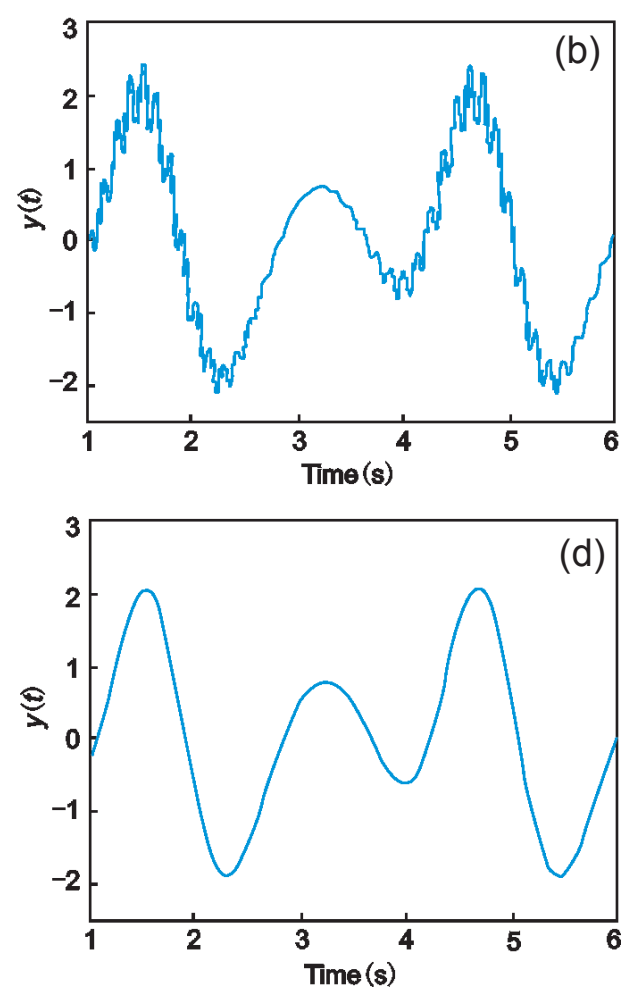

(a) unfiltered; (b) filtered by $9 \mathrm{~Hz}$; (c) filtered

2(a). It meant that the first type of frequency was not the desired cutoff frequency. Then the noise frequency of $7.96 \mathrm{~Hz}$ was adopted and the result curve was shown in Fig. 2(c). Although the amplitude of the fluctuations was decreased, the fluctuations of the curve were not eliminated. This made the noise frequency not suitable as the cutoff frequency. The last type of frequency 
was $7.7 \mathrm{~Hz}$, which was a bit lower than the noise frequency. In Fig. 2(d), a very smooth curve was found as the result. The fluctuations were completely removed. Therefore, the best cutoff frequency was $7.7 \mathrm{~Hz}$ in this case.

According to the discussion above, if the frequency of the noise can be determined, the best cutoff frequency for the low pass filtering method was a bit lower than the noise frequency. As shown in Fig. 3(a), the fluctuations of the temperature curves caused by the noise became obvious after $15 \mathrm{~s}$ when the temperature changes tended to be gentle. Figure $3(\mathrm{~b})$ shows the enlarged detail of the rectangular area designated by ' $a$ ' in Fig. 3(a). As shown in Fig. 3(b), the frequency of the noise of the temperature can be determined and the value was $47 \mathrm{~Hz}$. Based on the above discussed method of determining the cutoff frequency, $40 \mathrm{~Hz}$ was chosen as the cutoff frequency of the low pass filtering method based on FFT.

As the cutoff frequency was determined, the measured data were processed. Figure 3(c) shows the processed curves of the temperature. The curves were very smooth. The fluctuations of the noise were eliminated.
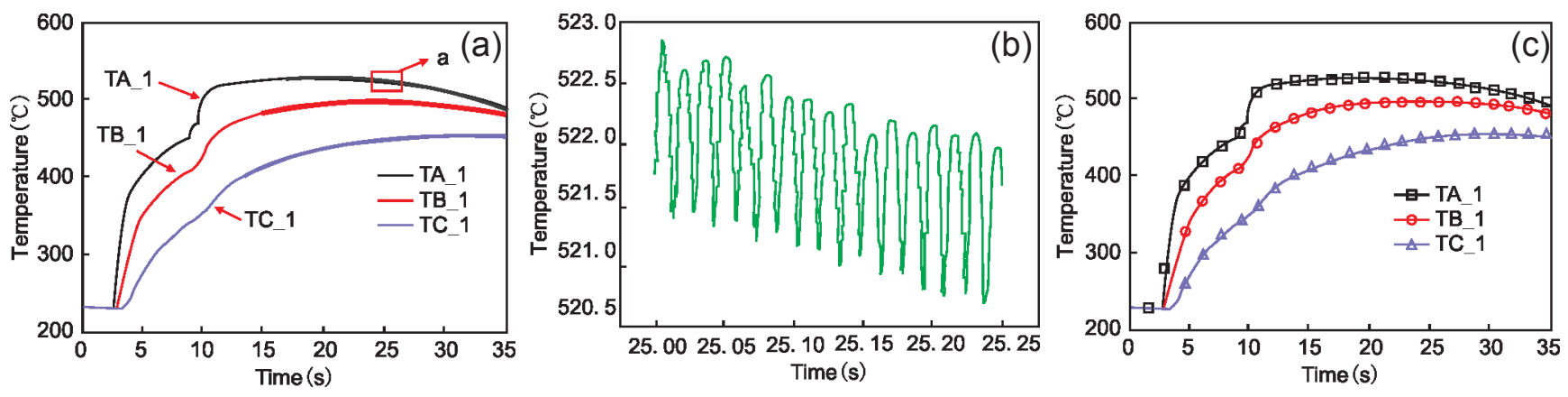

Fig. 3: Data processing: (a) original data; (b) enlarged detail of part 'a' in (a); (c) filtered data

\subsection{Mathematical modeling of IHTC}

The heat flux at the casting-die interface at each time-step is obtained by solving the inverse thermal problem ${ }^{[16]}$. The method introduced by Beck ${ }^{[17]}$ focused on minimizing the following function:

$$
\delta^{k}=\sum_{r=0}^{R-1}\left(Y^{k+r}-T^{k+r}\right)^{2}
$$

where $Y$ is the measured temperature, $T$ is the estimated temperature.

By the Taylor expansion, the solution of Eq. (2a) can be transformed into Eq. (2b):

$$
\Delta q^{k+1}=\frac{\sum_{r=0}^{R-1}\left(Y^{k+r}-T^{k+r}\right) \phi^{k+r}}{\sum_{r=0}^{R-1}\left(\phi^{k+r}\right)^{2}}
$$

where $R$ is the future time-step $R=r \Delta t, \phi$ is the sensitivity coefficient defined by Eq. (2c):

$$
\phi=\frac{\partial T}{\partial q}
$$

The heat flux of the next time-step $q^{k+1}$ could be obtained by correcting $q^{k}$ by the addition of $\Delta q^{k+1}$ :

$$
q^{k+1}=q^{k}+\Delta q^{k+1}
$$

This calculation procedure is repeated until the value of $\Delta q^{k+1} / q^{k+1}$ meets the requirement of $\Delta q^{k+1} / q^{k+1}<\varepsilon$.

Following the previous equations, the heat flux and the die surface temperature can be estimated by the inverse approach. The temperature inside the casting, especially the surface temperature must be estimated in order to evaluate the IHTC. The step center of the casting is adiabatic. Due to phase change of the casting, the heat transfer equation of the casting can be rewritten as Eq. (3):

$$
\rho\left[c-L \frac{\partial f_{s}}{\partial T}\right] \frac{\partial T}{\partial t}=\frac{\partial}{\partial x}\left(\lambda \frac{\partial T}{\partial x}\right)
$$

where $L$ is the latent heat of the casting, $f_{s}$ is the solid fraction in the casting. The term $\partial f_{s} / \partial T$ can be calculated by the solidification curve of the casting. Based on the determined heat flux and the temperature distributions inside the die and casting, the IHTC can be evaluated. The verification of the IHTC was discussed in Ref. [16].

\section{Results and discussion}

The following results were obtained under a casting pressure of $70 \mathrm{MPa}$. The pouring temperature and the initial die temperature were maintained at $933 \mathrm{~K}\left(660^{\circ} \mathrm{C}\right)$ and $503 \mathrm{~K}\left(230{ }^{\circ} \mathrm{C}\right)$, respectively prior to casting. The measured and simulated temperatures at $3 \mathrm{~mm}$ (TB) show a good agreement, and a maximum difference of about $278 \mathrm{~K}\left(5^{\circ} \mathrm{C}\right)$ was found between these two temperature profiles indicating that the determined results were quite reliable.

As shown in Fig. 4(a), the pouring point was designated by $\mathrm{V}$. The temperature of the die rose sharply at the beginning, then the temperature change became gentle. At the ninth second mark, the pressure of $70 \mathrm{MPa}$ was applied to the casting. Immediately after the pressure was applied, the temperatures designated by TA were increased sharply, and the other temperatures were also increased. Then, after reaching the maximum value, the temperatures decreased. The temperature maximum difference 
(a)

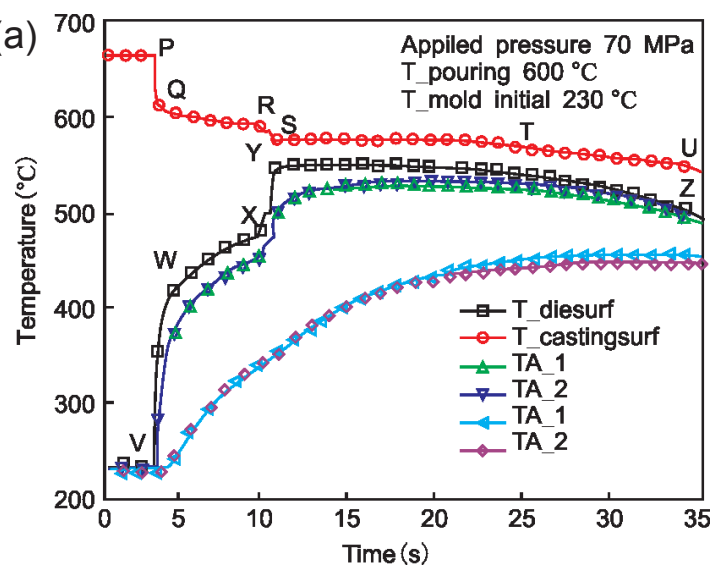

(b)

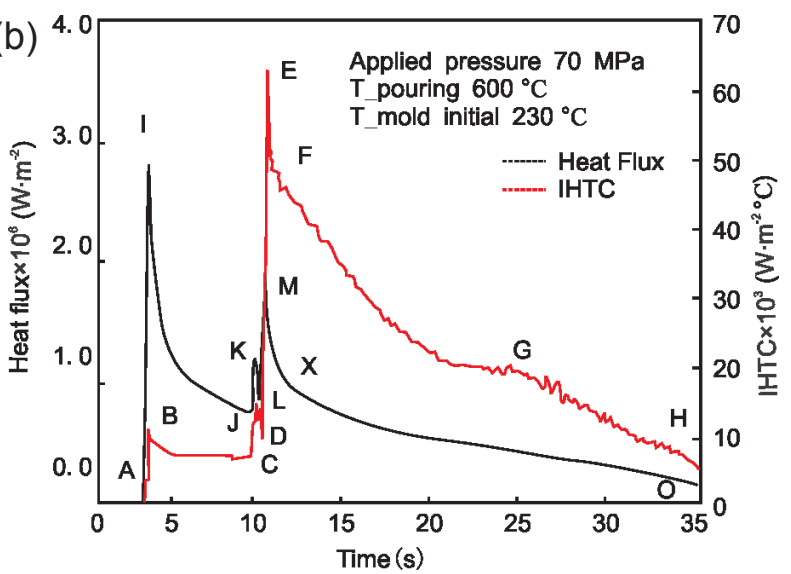

Fig. 4: Evaluation of IHTC: (a) measured die and casting surface temperature; (b) determined IHTC and heat flux at casting-die interface

of the symmetrically arranged thermocouples at $1 \mathrm{~mm}$ (TA_1 and TA_2) and $6 \mathrm{~mm}$ (TC_1 and TC_2) at Location 2, shown in Fig. 4(a), were $277 \mathrm{~K}\left(4{ }^{\circ} \mathrm{C}\right)$ and $279 \mathrm{~K}\left(6^{\circ} \mathrm{C}\right)$, respectively.

As shown in Fig. 4(a), the casting surface temperature and the die surface temperature were designated by T_castingsurf and T_diesurf. Segment PQ of T_castingsurf represented the period immediately after metal pouring. The sharp decrease of the casting surface temperature occurred at the beginning of metal solidification. Along with such decrease, there was a corresponding increase in the die surface temperature as designated by segment VW. Figure 4(b) shows the estimated heat flux and the IHTC of the casting with respect to the measured temperature profiles shown in Fig. 4(a). Corresponding to the rapid decrease of the casting surface temperature (segment PQ), the heat flux, designated by segment AI, rose sharply as soon as the high temperature melt was poured into the cavity until reaching a peak value of about 2.84 $\mathrm{MW} \cdot \mathrm{m}^{-2}$. The IHTC, designated by segment $\mathrm{AB}$, also increased to a value of about $10.58 \mathrm{~kW} \cdot \mathrm{m}^{-2} \cdot \mathrm{K}^{-1}$.

After segment $\mathrm{PQ}$, the casting surface temperature was followed by a continuous decrease designated by the segment QR. After reaching the peak value, the heat flux, designated by segment IJ, dropped rapidly until its value was about $0.7 \mathrm{MW} \cdot \mathrm{m}^{-2}$. At the same time, the IHTC also decreased to $6.6 \mathrm{~kW} \cdot \mathrm{m}^{-2} \cdot \mathrm{K}^{-1}$ and lasted for about $4 \mathrm{~s}$.

At the ninth second mark, pressure was applied on the casting. Immediately after the pressure was applied, the casting surface temperature abruptly decreased as designated by segment RS in Fig. 4(a). Corresponding with segment RS, segment XY presents the sharply increased die surface temperature. As soon as the pressure was applied, the IHTC abruptly increased to the peak value of about $62.8 \mathrm{~kW} \cdot \mathrm{m}^{-2} \cdot \mathrm{K}^{-1}$, designated by segment DE, as shown in Fig. 4(b). It indicated that the closest contact between the casting and die was achieved in this experiment as the pressure was applied.

After the pressure application came the pressure maintaining stage, the die temperature decreased gradually. The temperature difference between the casting surface and the die surface increased gradually. This indicated that the solidification was slowly coming to the end. As shown in Fig. 4(b), the IHTC, designated by segment EFG, sharply dropped at first. Then, the decrease rate of the IHTC became lower and lower. At the end, the IHTC was nearly unchanged until it approached Point G. After that, the IHTC decreased again. This meant that the applied pressure had a great influence on the IHTC before the moment represented by Point G. After that moment, the decrease of the IHTC may be caused by the formation of the gap at the casting-die interface.

\section{Conclusions}

In order to obtain accurate IHTC, a series of squeeze casting experiments were conducted and a "plate shape" aluminum alloy A356 casting was cast in $\mathrm{H} 13$ steel die.

(1) A special temperature sensor unit was designed to overcome the installation difficulties and to solve the problem of how to accurately estimate the IHTC under the condition that the heat flux at the casting-die interface increases dramatically.

(2) In order to eliminate the negative effect of the highfrequency noise components on the analysis of the IHTC, a low pass filtering method based on Fast Fourier Transform (FFT) was adopted to process the data.

(3) The IHTC at the casting-die interface rose to the peak value almost simultaneously as soon as the pressure was applied. The peak value of the IHTC of the casting was $62.8 \mathrm{~kW} \cdot \mathrm{m}^{-2} \cdot \mathrm{K}^{-1}$. There was a pressure maintaining stage immediately after the pressure was applied. During this stage, the IHTC sharply dropped at first. Then, the decrease rate of the IHTC became lower and lower. At last, the IHTC was nearly unchanged until it approached Point G. After that, the IHTC decreased again. This meant that the applied pressure had a great influence on the IHTC before the moment represented by Point G. After that moment, the decrease of the IHTC may be caused by the formation of the gap at the casting-die interface.

\section{References}

[1] El-khair M T A. Microstructure characterization and tensile properties of squeeze-cast AlSiMg alloys. Mater. Lett., 2005, 59: 894-900. 
[2] Su J Q, Nelson T W, Mishra R, et al. Microstructural investigation of friction stir welded 7050-T651 aluminium. Acta. Mater., 2003, 51: 713-729.

[3] Singh R K R, Sharma C, Dwivedi D K, et al. The microstructure and mechanical properties of friction stir welded Al-Zn-Mg alloy in as welded and heat treated conditions. Mater. Des., 2011, 32: 682-687.

[4] Cao Y Y, Guo Z P and Xiong S M. Determination of the metal/ die interfacial heat transfer coefficient of high pressure die cast B390 alloy. IOP Conference Series: Materials Science and Engineering, 2012.

[5] Yue T M and Chadwick G A. Squeeze casting of light alloys and their composites. J. Mater. Process. Tech., 1996, 58:302-307.

[6] Zhang D L, Cantor B. A numerical heat flow model for squeeze casting Al alloys and Al alloy/SiCp composites. Modelling and Simulation in Materials Science and Engineering, 1995, 3(1):121-130.

[7] Lee J H, Kim H S, Won C W. Effect of the gap distance on the cooling behavior and the microstructure of indirect squeeze cast and gravity die cast 5083 wrought Al alloy. Mater. Sci. Eng. A., 2002, 338(1-2):182-190.

[8] Han Z Q, Zhu W, and Liu B C. Thermomechanical modeling of solidification process of squeeze casting I. Mathematic Model and Solution Methodology. Acta. Metall. Sin., 2009, 45: 356362.

[9] Tang J, Han Z Q, Wang F F, et al. A Coupled Thermo-Mechanical Simulation on Squeeze Casting Solidification Process of ThreeDimensional Geometrically Complex Components. Advances in the Science and Engineering of Casting Solidification, 2015: 113-120.
[10] Zhu W, Han Z Q, and Liu B C. Thermomechanical modeling of solidification process of squeeze casting II. Numerical Simulation and Experimental Validation. Acta. Metall. Sin, 2009, 45: 353-368.

[11] Aweda J O and Adeyemi M B. Determination of temperature distribution in squeeze cast aluminum using the semi-empirical equations method. J. Mater. Process. Tech., 2009, 209(17): 5751-5759.

[12] Aweda J O and Adeyemi M B. Experimental determination of heat transfer coefficients during squeeze casting of aluminum. J. Mater. Process. Tech., 2009, 209(3): 1477-1483.

[13] Ilkhchy A F, Jabbari M, and Davam P. Effect of pressure on heat transfer coefficient at the metal/mold interface of A356 aluminum alloy. Int. Commun. Heat. Mass., 2012, 39(5): 705-712.

[14] Sun Z, Hu H, and Niu X. Determination of heat transfer coefficients by extrapolation and numerical inverse methods in squeeze casting of magnesium alloy AM60. J. Mater. Process. Tech., 2011, 211(8): 1432-1440.

[15] Sun Z, Hu H, and Niu X. Experimental study and numerical verification of heat transfer in squeeze casting of aluminum alloy A443. Metall. Mater. Trans. B, 2012, 43B: 1676-1683.

[16] Wang F F, Ma Q X, Meng W, et al. Experimental study on the heat transfer behavior and contact pressure at the casting-mold interface in squeeze casting of aluminum alloy. Int. J. Heat. Mass. Tran., 2017, 112: 1032-1043.

[17 Beck J V. Non-linear estimation applied to the nonlinear inverse heat conduction problem. Int. J. Heat. Mass. Tran., 1970, 13: 703-716. 